PREPARED FOR THE U.S. DEPARTMENT OF ENERGY, UNDER CONTRACT DE-AC02-76CH03073

PPPL-3558

PPPL-3558

UC-70

Reduction of Islands in Full-pressure Stellarator Equilibria

by

S.R. Hudson, D.A. Monticello, and A.H. Reiman

April 2001

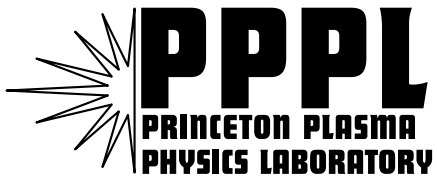

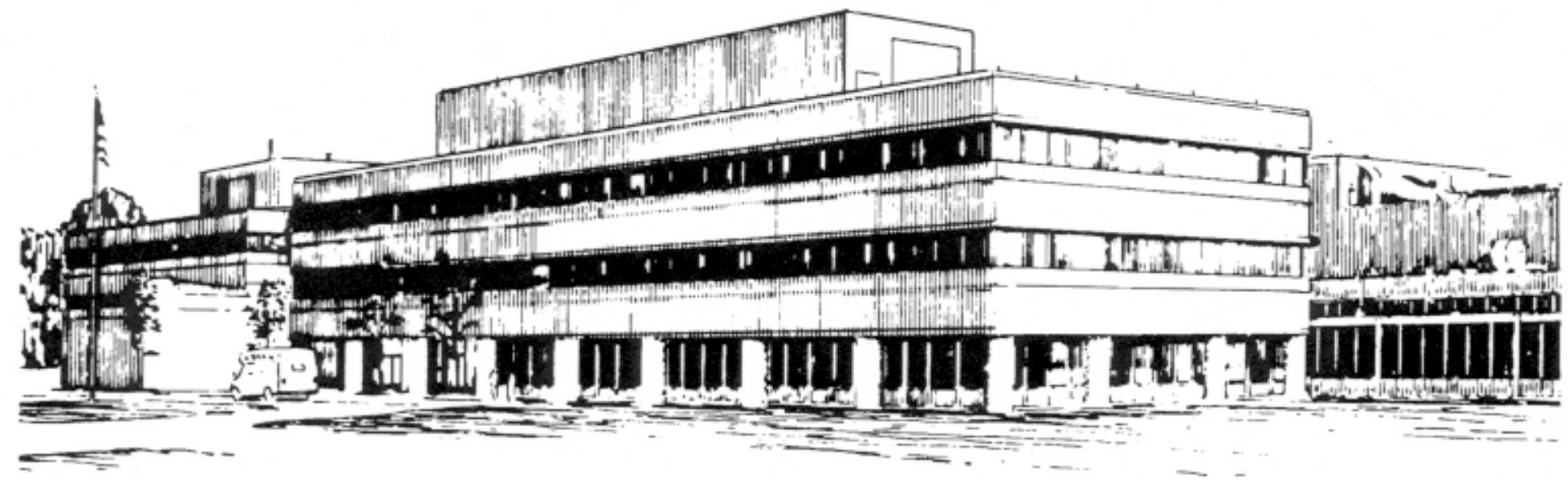

PRINCETON PLASMA PHYSICS LABORATORY PRINCETON UNIVERSITY, PRINCETON, NEW JERSEY 


\section{PPPL Reports Disclaimer}

This report was prepared as an account of work sponsored by an agency of the United States Government. Neither the United States Government nor any agency thereof, nor any of their employees, makes any warranty, express or implied, or assumes any legal liability or responsibility for the accuracy, completeness, or usefulness of any information, apparatus, product, or process disclosed, or represents that its use would not infringe privately owned rights. Reference herein to any specific commercial product, process, or service by trade name, trademark, manufacturer, or otherwise, does not necessarily constitute or imply its endorsement, recommendation, or favoring by the United States Government or any agency thereof. The views and opinions of authors expressed herein do not necessarily state or reflect those of the United States Government or any agency thereof.

\section{Availability}

This report is posted on the U.S. Department of Energy's Princeton Plasma Physics Laboratory Publications and Reports web site in Calendar Year 2001. The home page for PPPL Reports and Publications is: http://www.pppl.gov/pub_report/

DOE and DOE Contractors can obtain copies of this report from:

U.S. Department of Energy

Office of Scientific and Technical Information

DOE Technical Information Services (DTIS)

P.O. Box 62

Oak Ridge, TN 37831

Telephone: (865) 576-8401

Fax: (865) 576-5728

Email: reports@adonis.osti.gov

This report is available to the general public from:

National Technical Information Service

U.S. Department of Commerce

5285 Port Royal Road

Springfield, VA 22161

Telephone: 1-800-553-6847 or

(703) 605-6000

Fax: (703) 321-8547

Internet: http://www.ntis.gov/ordering.htm 


\title{
Reduction of islands in full-pressure stellarator equilibria.
}

\author{
S.R.Hudson, D.A.Monticello and A.H.Reiman \\ Princeton Plasma Physics Laboratory, PO Box 451 Princeton NJ 08543 \\ Physics of Plasmas, 2001
}

April 24, 2001

\begin{abstract}
The control of magnetic islands is a crucial issue in designing stellarators. Islands are associated with resonant radial magnetic fields at rational rotational-transform surfaces and can lead to chaos and poor plasma confinement. In this article we show that variations in the resonant fields of a full pressure stellarator equilibrium can be related to variations in the boundary via a coupling matrix, and inversion of this matrix determines a boundary modification for which the island content is significantly reduced. The numerical procedure is described and the results of island optimization are presented. Equilibria with islands are computed using the Princeton Iterative Equilibrium Solver, and resonant radial fields are calculated via construction of quadratic-flux-minimizing surfaces. A design candidate for the National Compact Stellarator Experiment [Phys.Plasmas 8,2001.], which has a large island, is used to illustrate the technique. Small variations in the boundary shape are used to reduce island size and to reverse the phase of a major island chain.
\end{abstract}

\section{Introduction}

The suppression of magnetic islands is a key issue in stellarator designs. The lack of a continuous symmetry implies that magneto-hydro-dynamic (MHD) equilibrium solutions typically will have magnetic islands and chaotic field lines; however, with careful design, the width of islands and chaotic regions may be significantly reduced. Significant progress in this field of island suppression was made with the introduction of the Cary-Hanson technique [1], which relates the width of magnetic islands to the residue of periodic orbits determined by field line integration, and standard computational optimization routines can be used efficiently to construct coil configurations that produce nested flux surfaces in the vacuum. An alternative technique [2] has been applied to the H-1 Heliac [3]. Resonant radial fields were determined via construction of quadratic-flux-minimizing surfaces and variations in the vertical field coil currents were used to manipulate the width and phase of magnetic islands in the vacuum field. A configuration was constructed for which vacuum magnetic islands healed as pressure was increased [4].

In this article we consider manipulation of the width and phase of magnetic islands in finite $\beta$ stellarator equilibria by making small variations to the boundary. Computation of the MHD equilibrium is provided by the Princeton Iterative Equi- librium Solver, PIES [5, 6]. Magnetic islands are controlled by controlling the resonant fields at the rational surfaces, and the resonant fields are calculated via construction of quadraticflux-minimizing surfaces [7]. In Sec. II a description of the computational tools is given, and in Sec. III the numerical procedure is described. A design candidate for the National Compact Stellarator Experiment, NCSX [8, 9] is used to illustrate the island manipulation procedure and results are presented in Sec. IV.

\section{Resonant Fields and PIES}

The PIES code finds solutions to the magneto-hydro-dynamic equilibrium condition $\nabla p=\mathbf{J} \times \mathbf{B}$ for general stellarator geometry using an iteration procedure. The equilibrium is defined by a toroidal current and pressure profile, and a boundary shape. An approximate equilibrium is used to start the PIES iterations and this is usually provided by the Variation Moments Equilibrium Code, VMEC [10]. VMEC calculates solutions to the MHD equilibrium condition assuming the existence of good flux surfaces. The boundary is specified by a set of Fourier harmonics for the cylindrical coordinates $\mathrm{R}$ and $\mathrm{Z}$ in the cylindrical toroidal angle and a poloidal angle. We expect that the VMEC equilibrium contains singular current sheets at the rational surfaces - the magnitude of which is sufficient to suppress suppress island formation.

The PIES procedure involves relaxing the constraint of good flux surfaces of the magnetic field as calculated by VMEC, and iterates on the field and current using a Picard Scheme. The current is calculated according to $\nabla p=\mathbf{J}^{n+1} \times \mathbf{B}^{n}$ with the constraint $\nabla \cdot \mathbf{J}^{n+1}=0$. The magnetic field is updated using $\mathbf{J}^{n+1}=\nabla \times \mathbf{B}^{n+1}$ with the constraint $\nabla \cdot \mathbf{B}^{n+1}=0$. At each iteration, a magnetic field diagnostic distinguishes regions of good flux surfaces and regions of islands and chaotic field lines. On good flux surfaces magnetic coordinates are used to solve a magnetic differential equation for the parallel current. In islands and chaotic regions the pressure and current profiles are flattened. On convergence, the equilibrium condition $\nabla p=$ $\mathbf{J} \times \mathbf{B}$ is satisfied and in general islands will exist at the rational surfaces; thus resolving the current singularities in the VMEC equilibrium. If the VMEC equilibrium is such that the current singularities are small or zero, then we expect that the island width as calculated by PIES will be small or zero, that the VMEC and PIES equilibriums will agree, and that PIES will rapidly converge. In the following sections, each separate PIES calculation is initialized with a VMEC calculation. 
Magnetic islands are caused by resonant radial magnetic fields where the rotational transform is a rational value. The continuous one-dimensional family of periodic orbits that form a rational rotational transform flux surface in the absence of resonant fields will be reduced to a finite set of periodic orbits by the resonant field, and an island chain will form. The periodic orbits surviving perturbation will typically be the stable and unstable periodic orbits, which correspond to the $\mathrm{O}$ and $\mathrm{X}$ points on Poincaré plots of the magnetic field. In the small island approximation, where the shear, $t^{\prime}$, is assumed constant across the island, the width of the island is given [11] as $w \propto \sqrt{\left|B_{n m}\right| / t^{\prime} m}$, where $B_{n m}=(\mathbf{B} \cdot \nabla s / \mathbf{B} \cdot \nabla \phi)_{n m}$ is the resonant Fourier component of the radial field at the $t=n / m$ rational surface, $s$ is the radial coordinate, and the prime represents derivative with respect to $s$. The phase of the island chain is determined by the sign of $B_{n m}$ and the sign of the shear. The manipulation of island width and phase presented in the following is enabled via control of the magnitude and sign of the resonant field.

A method for calculating resonant fields at rational surfaces has been incorporated into PIES. This method is based on the construction of quadratic-flux-minimizing surfaces. The construction of these surfaces has been presented earlier [7] and for the purposes of this article it is sufficient to note that a rational quadratic-flux-minimizing surface passes directly through the corresponding island chain, and may be considered as a rational flux surface of an underlying unperturbed magnetic field. The resonant radial field is constructed as the field normal to the quadratic-flux-minimizing surface. In the following, the term 'resonant field' shall refer to the action gradient [2] as calculated during the construction of a given quadratic-fluxminimizing surface.

\section{Coupling Matrix}

A set of islands that we wish to control is selected. Generally the lowest order resonances present will produce the largest magnetic islands. A convenient method of selecting the lowest order rationals is guided by the Farey Tree construction [12]. The corresponding set of resonant fields that need to be controlled is represented by $\mathbf{B}=\left(B_{n_{1} m_{1}}, B_{n_{2} m_{2}}, \ldots\right)^{T}$.

We expect that an $(n, m)$ island width will be strongly affected by an $(n, m)$ resonant deformation of the plasma boundary in magnetic coordinates and perhaps through coupling to neighboring modes, so a set of independent boundary variation parameters is constructed as follows. We consider the minor radius $r=\sum r_{n m} \cos (m \theta-n N \phi)$ of the plasma boundary to be a Fourier series in the cylindrical toroidal angle and the poloidal angle used in VMEC to construct the input $R$ and $Z$ harmonics. The conversion to cylindrical space is given as $R=r \cos \theta, Z=r \sin \theta$. For a change $r \rightarrow r+\delta r_{n m} \cos (m \theta-n N \phi)$, the input Fourier harmonics for the VMEC code change according to $R_{m-1, n} \rightarrow$ $R_{m-1, n}+\delta r_{n m} / 2, R_{m+1, n} \rightarrow R_{m+1, n}+\delta r_{n m} / 2, Z_{m-1, n} \rightarrow$ $R_{m-1, n}-\delta r_{n m} / 2, Z_{m+1, n} \rightarrow R_{m+1, n}+\delta r_{n m} / 2$. In principle we may change infinitely many boundary harmonics $r_{n m}$, but a small set is chosen to match the islands that will be targeted and this becomes the vector of independent parameters $\mathbf{r}=\left(r_{n_{1} m_{1}}, r_{n_{2} m_{2}}, \ldots\right)^{T}$.
Now the problem is amenable to standard treatments where the functional dependence of $\mathbf{B}$ on $\mathbf{r}$ is represented

$$
\mathbf{B}\left(\mathbf{r}_{0}+\delta \mathbf{r}\right)=\mathbf{B}\left(\mathbf{r}_{0}\right)+\mathbf{C} \cdot \delta \mathbf{r}+\ldots,
$$

where $\mathbf{r}_{0}=0$ is the initial boundary shape and $\delta \mathbf{r}$ is a small boundary variation. The coupling matrix $\mathbf{C}$ represents derivative information and will in general be an $M \times N$ matrix, where $M$ is the number of resonant fields, and $N$ is the number of independent boundary variations. The $j t h$ column of the coupling matrix is determined through a VMEC/PIES run by making a small change $\delta r_{n_{j} m_{j}}$ and taking the difference in the resonant fields from the original equilibrium, divided by the change. Hence, $N+1 \mathrm{VMEC} / \mathrm{PIES}$ runs are required to determine the coupling matrix.

The coupling matrix is inverted using the singular value representation [13], $\mathbf{C}=\mathbf{U} \mathbf{w}^{T}$, where $\mathbf{U}$ and $\mathbf{V}$ are orthonormal and $\mathbf{w}$ is the diagonal matrix of singular values. If there are more variables than equations more than one solution may exist and the nullspace is spanned by the columns of $U$ corresponding to zero singular values, of which there will be at least $N-M$.

Islands are removed if $\mathbf{B}=\mathbf{0}$, so by choosing a correction to the boundary $\delta \mathbf{r}$ according to

$$
\delta \mathbf{r}_{i+1}=-\mathbf{V} \mathbf{w}^{-1} \mathbf{U}^{T} \mathbf{B}_{i},
$$

where as in standard singular value decomposition techniques the zero, and if desired the small, eigenvalues are ignored in the inversion of $\mathbf{w}$, and $\mathbf{B}_{i}$ is the vector of resonant fields at the ith iteration. In practice, several iterations will be required to achieve a desired accuracy.

\section{Application to NCSX}

To illustrate the technique a design candidate, li383, for NCSX is studied. The motivation of the NCSX design group is to achieve compact stellarator configurations with good transport and stability properties at $\beta=4 \%$, with quasi-axisymmetry used to obtain good drift trajectories. Cross sections of the configuration li383 are shown in Fig. 1, and the rotational transform profile is shown in Fig. 2. A Poincaré plot Fig. 3 of the PIES field after 32 iterations shows island chains and the $t=3 / 5$ island is quite large. In this and the other Poincaré plots to be shown, the Poincaré section is the $\phi=0$ plane and 50 field lines are followed starting along the $\theta=0$ line. In addition, field lines at the $\mathrm{X}$ points of several low order island chains are followed and the quadratic-flux minimizing surface and an estimated separatrix has been plotted over one period of each island chain. The separatrix of the island chains has been calculated using the resonant radial field and the shear at the rational surface of the VMEC equilibrium. PIES has not yet converged for this case, but the information about the island width is still useful for construction of the coupling matrix.

In this application of the island reducing technique, the $(3,5),(6,10),(3,6)$ and $(6,12)$ resonances are targeted, and the $(3,9),(3,8),(3,7),(3,6),(3,5)$ and $(3,4)$ boundary harmonics are varied. The $(3,7)$ resonance is also present in the configuration, but this has not been targeted. The $(6,10)$ resonance 


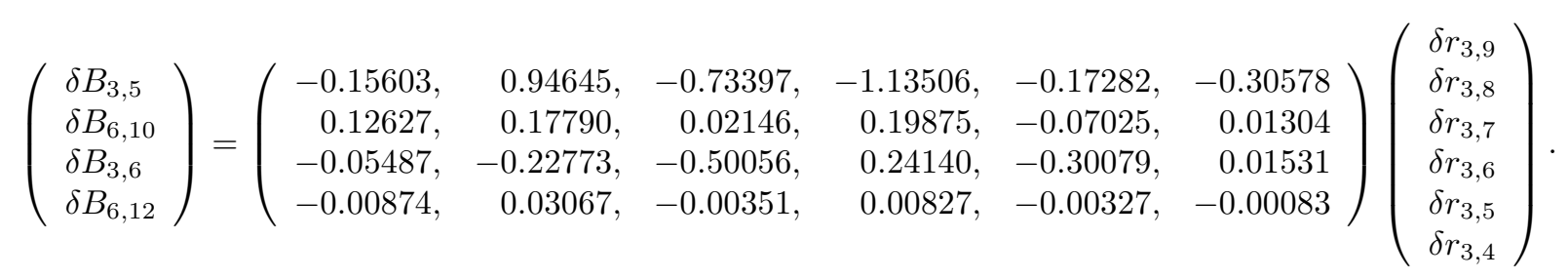

Figure 1: Cross sections of NCSX design li383.

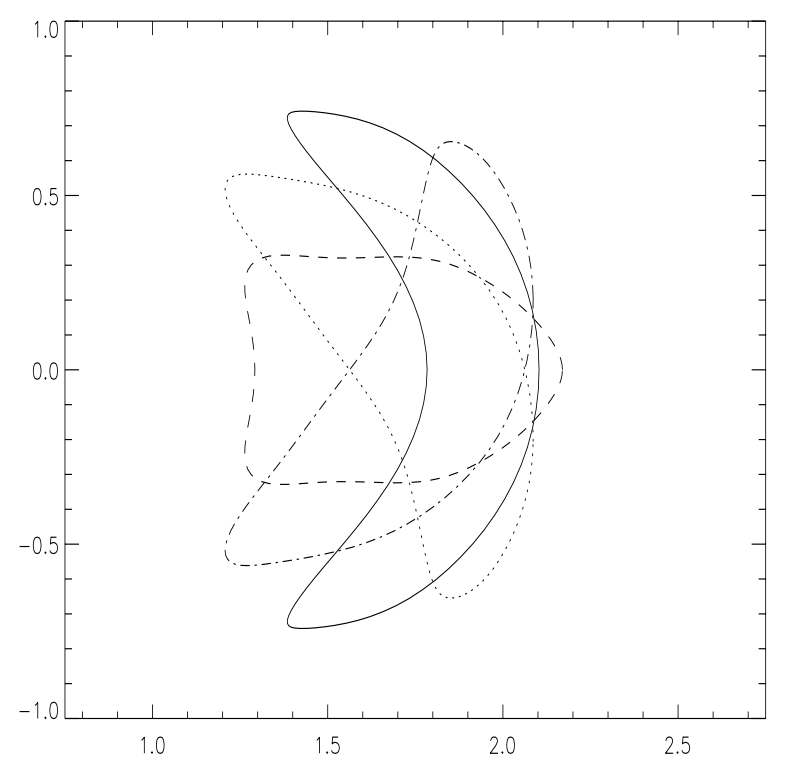

Figure 3: Poincaré plot of full-beta initial li383 configuration after 32 PIES iterations.

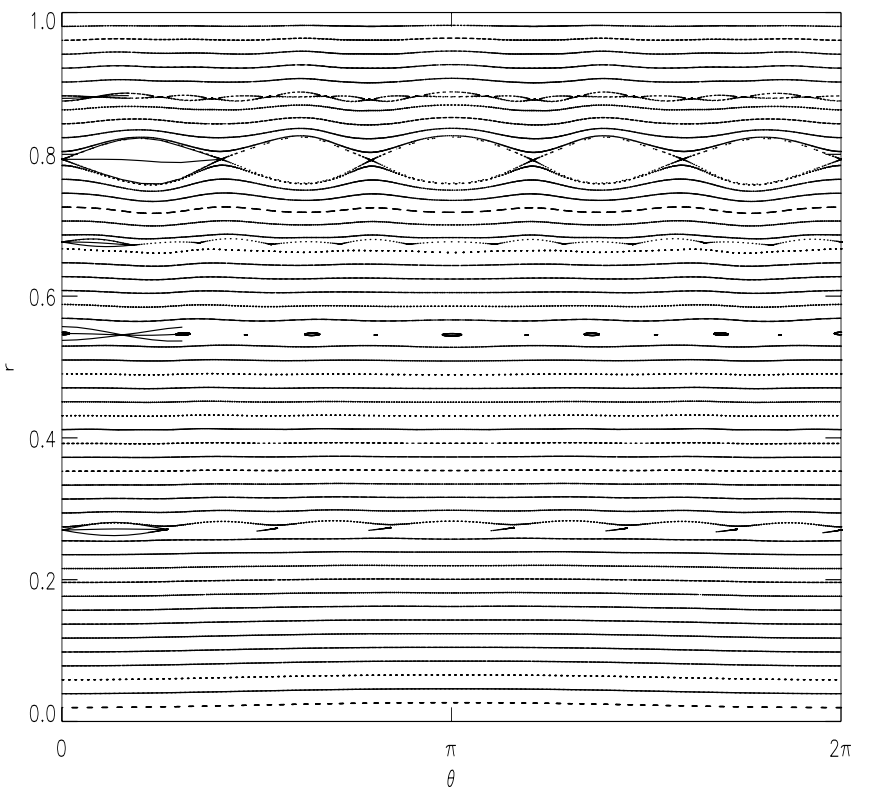

Table 1: Convergence of Newton Method

Figure 2: Rotational transform profile of the li383 equilibrium.

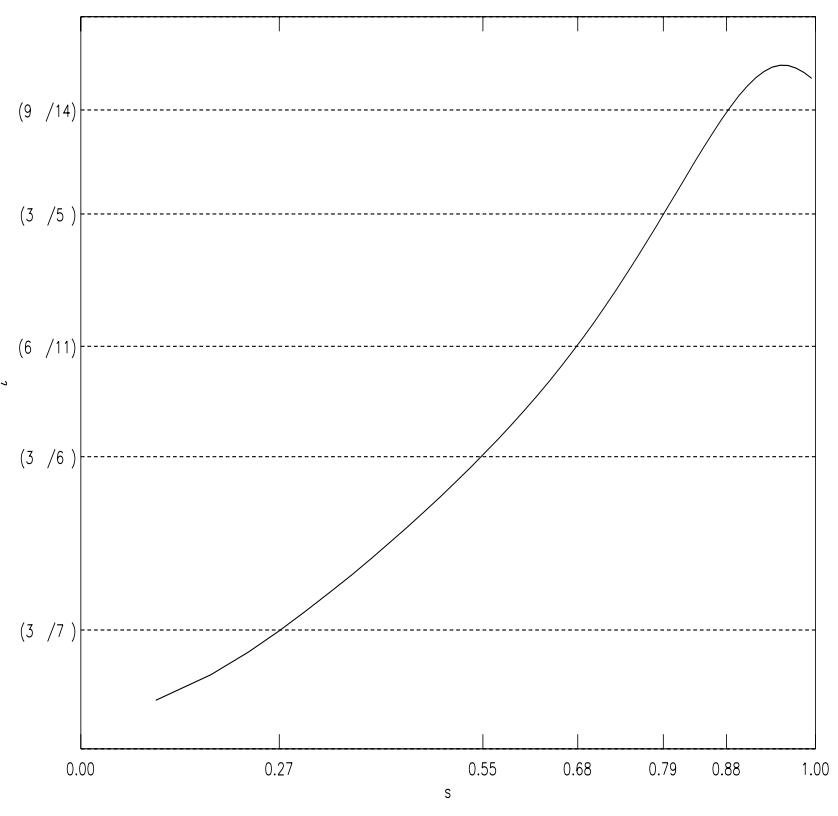

\begin{tabular}{ccccc}
\hline \hline iteration & $\left|B_{3,5}\right|$ & $\left|B_{6,10}\right|$ & $\left|B_{3,6}\right|$ & $\left|B_{6,12}\right|$ \\
\hline 0 & $1.8 \times 10^{-3}$ & $1.6 \times 10^{-4}$ & $1.3 \times 10^{-4}$ & $1.4 \times 10^{-5}$ \\
1 & $1.3 \times 10^{-4}$ & $3.4 \times 10^{-5}$ & $1.0 \times 10^{-4}$ & $2.4 \times 10^{-6}$ \\
2 & $6.7 \times 10^{-5}$ & $3.4 \times 10^{-5}$ & $5.1 \times 10^{-5}$ & $1.9 \times 10^{-6}$ \\
3 & $2.4 \times 10^{-5}$ & $6.7 \times 10^{-5}$ & $4.0 \times 10^{-6}$ & $5.4 \times 10^{-7}$ \\
\hline \hline
\end{tabular}

produces an island at the same rational surface as the $(3,5)$, namely at $t=3 / 5$, and may be considered as the second harmonic of the $(3,5)$ resonance. If the $(6,10)$ resonant field is not targeted, this may cause an island of distinct topology from the $(3,5)$. For this set of resonant fields and independent boundary variation parameters, the coupling matrix is shown in Eqn(3). Table. 1 details the convergence of the Newton iterations. The Newton iterations are terminated after four steps as this provides sufficient reduction of the islands as seen in Fig. 4. In a true Newton iteration procedure, the coupling matrix would be re-calculated at every iteration. In this application such a procedure is too slow and the coupling matrix is not changed; nevertheless, the convergence is satisfactory. The total change 
Figure 4: Poincaré plot of full-beta island-reduced li383 configuration.

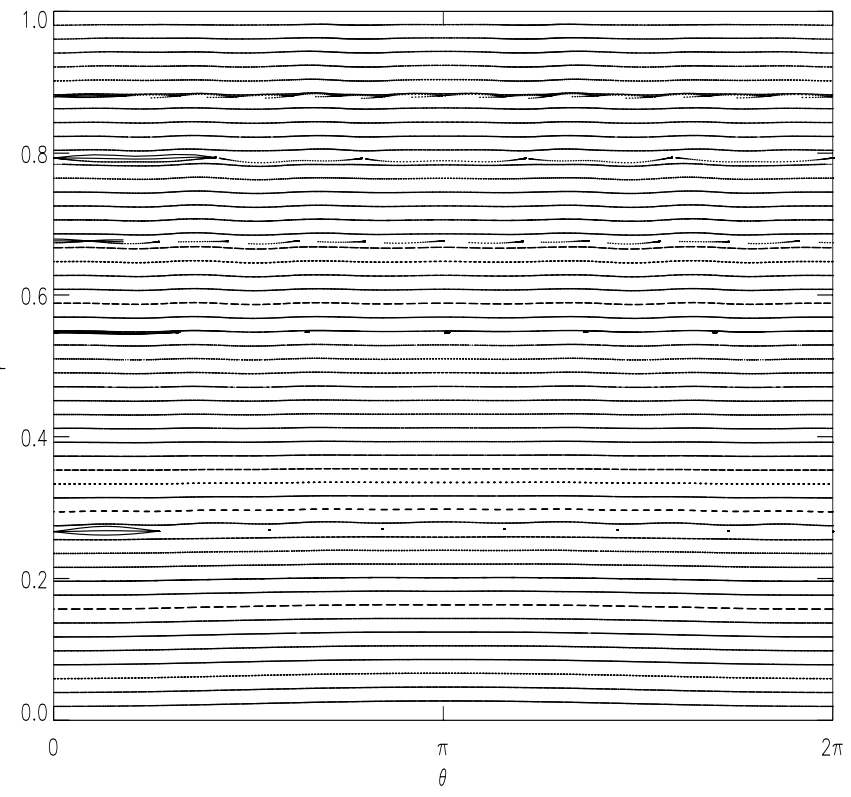

in the boundary variation parameters is

$$
\delta \mathbf{r}=(-0.0018,-0.0002,0.0005,0.0030,0.0001,0.0006)^{T} .
$$

These variations are several millimeters in magnitude and are too small to distinguish when plotted on the scale shown in Fig. 1, and generally have little impact on stability and other physics. However, the case shown does destabilize the ballooning modes on some surfaces. This is not surprising considering that the li383 configuration has been optimized to provide marginal ballooning stability at full pressure.

The healed configuration has converged after 32 iterations. As mentioned, if the equilibrium has no islands, or if the width of the islands is less than the radial grid used in PIES, then PIES and VMEC will agree and PIES will rapidly converge. If the equilibrium displays islands and PIES is not fully converged, the island width after a given number of iterations is still sufficient for calculation of the coupling matrix and for the success of the Newton procedure in removing islands.

The same procedure that is used to eliminate the islands may be used to create islands. With the possibility of self-healing effects and for the design of trim coils, where the flexibility to eliminate islands caused by design or construction error, which may be of either phase, is required, it is interesting to design configurations with islands of opposite phase. To do this, Eq. ( 2 becomes

$$
\delta \mathbf{r}=-\mathbf{V} \mathbf{w}^{-1} \mathbf{U}^{T}\left(\mathbf{B}-\mathbf{B}^{s}\right),
$$

where $\mathbf{B}^{s}$ is a vector of desired settings of the resonant fields. We choose to set $\mathbf{B}^{s}=\left(-B_{3,5}^{o}, 0,0,0\right)$, where $B_{3,5}^{o}$ is the resonant field component as determined for the original li383 configuration shown in Fig. 3, and proceed as before. Applying the boundary variation

$$
\delta \mathbf{r}=(-0.0041,-0.0036,0.0017,0.0042,0.0012,0.0013)^{T}
$$

to the original configuration swaps the phase of the $(3,5)$ island in the full pressure full current equilibrium shown in Fig. 5 . As

Figure 5: Poincaré plot of full-beta phase-reversed li383 configuration after 32 PIES iterations.

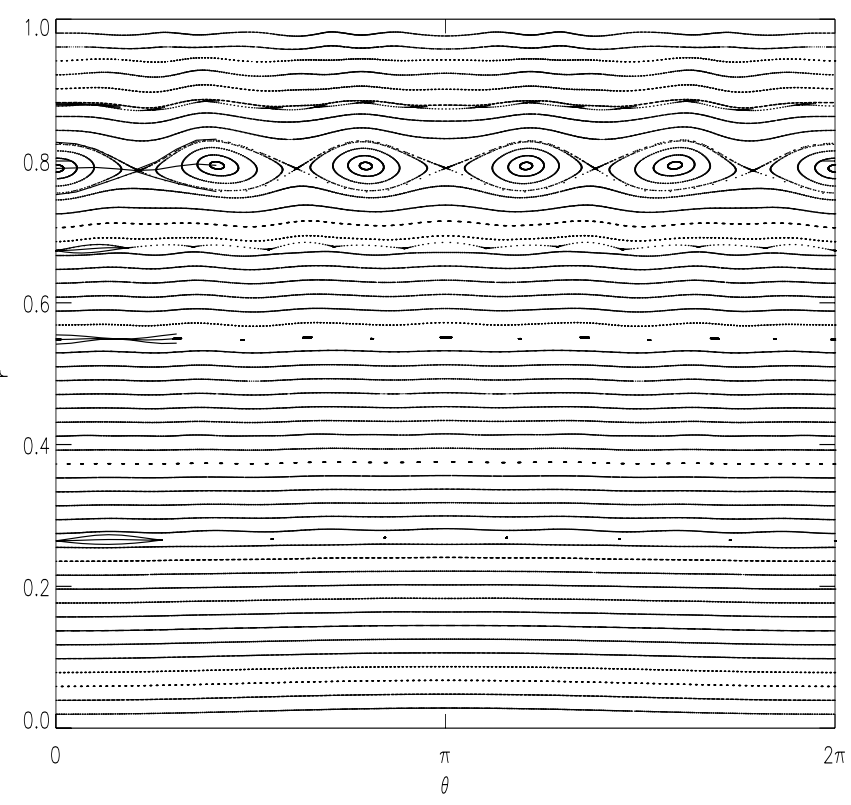

noted earlier, the phase of the island depends on the sign of the resonant field and the sign of the shear at the rational surface. The changes made to the equilibrium have no significant impact on the rotational transform profile; thus to change the phase of the island it is sufficient to control the resonant field only.

A zero-pressure equilibrium is calculated with PIES with the same boundary as the full pressure healed configuration. As pressure is lowered the islands reappear Fig. 6. To eliminate the islands at both zero and full pressure, use is made of the null-space of the coupling matrix. To the accuracy of the determination of the coupling matrix, any changes to the boundary which are spanned by the two columns of $\mathbf{V}$ corresponding to the zero eigenvalues of $\mathbf{w}$ will not impact on the island content of the full pressure healed configurations. Thus, to eliminate islands at both zero and full pressure, we make small changes within this nullspace to eliminate the islands at zero pressure. Another, reduced coupling matrix, of dimensions $4 \times 2$ is constructed, where the independent parameters are boundary variations spanned by the two nullspace vectors and the target variables are the same 4 resonant fields as targeted above. In such a case with more equations to be solved than variables, rather than being a family of solutions there is in fact no exact solution. In such a case the SVD procedure will provide an approximate solution and the island widths at zero pressure are reduced Fig. 7. Another PIES calculation Fig. 8 confirms that the zero pressure island healing variation do not disturb the full pressure islands. Again, these configurations, having small islands, are converged. 
Figure 6: Poincaré plot of full-beta island-reduced li383 configuration at zero beta.

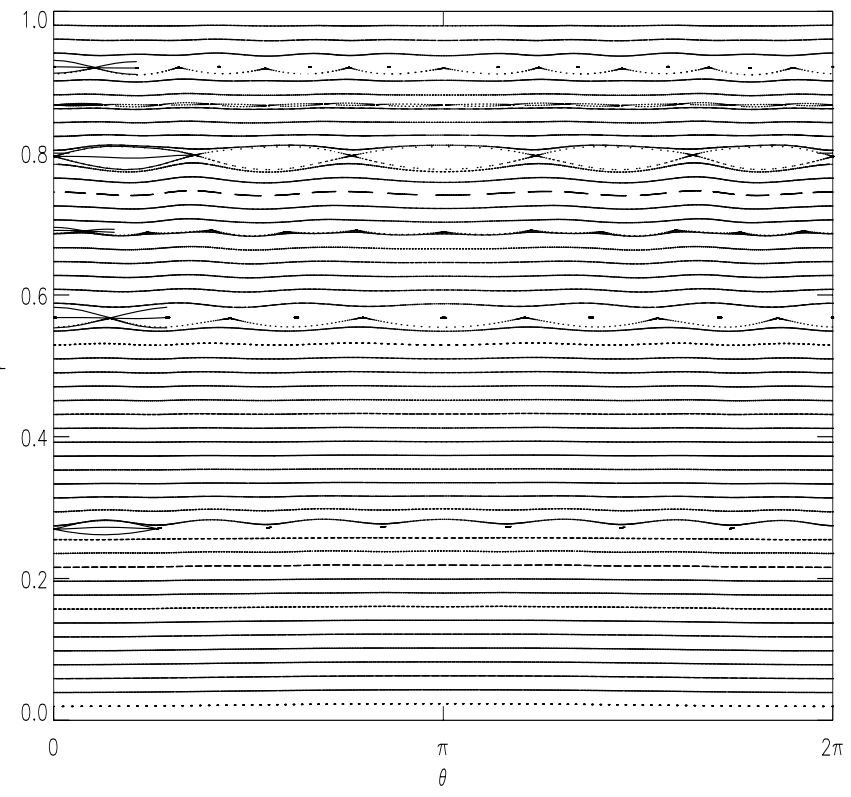

Figure 7: Poincaré plot of full-beta and zero-beta island reduced configuration at zero beta.

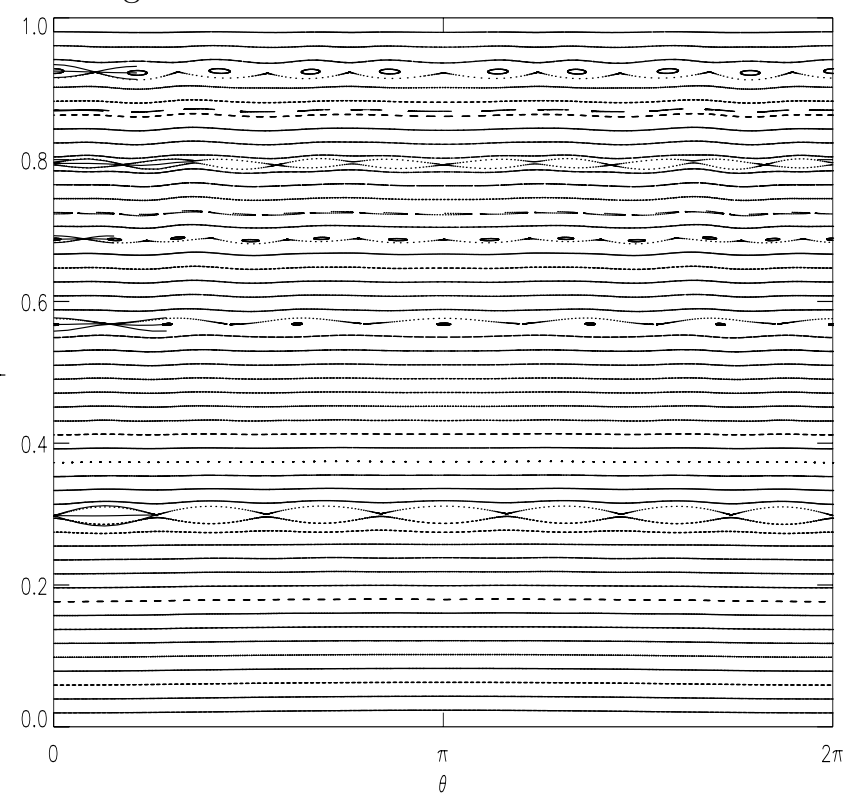

\section{Comments and Future Work}

Fixed boundary variations enable control of island phase and width. We have shown that a major island chain in the NCSX design candidate li383 my be eliminated or created with either phase by careful choice of boundary variation. In practice, the plasma boundary shape is controlled by the coil design and possibly by additional trim coils. With several independent boundary variation parameters, chosen to provide suffi-
Figure 8: Poincaré plot of full-beta and zero-beta island reduced configuration at full beta.

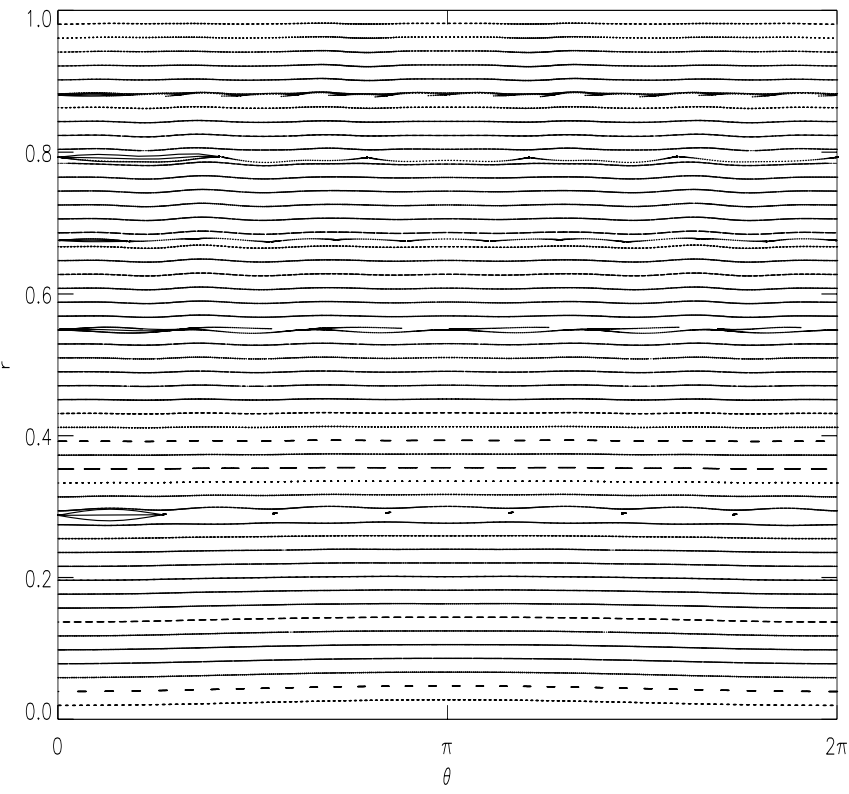

cient flexibility in controlling specific islands, several different plasma states may be optimized with respect to island reduction.

The fixed boundary variations required to eliminate the magnetic islands may be related to normal magnetic fields at the surface and from consideration of the coupling matrix it can be determined which fields generated by the coils are most crucial in reducing island widths and thus which aspects of the coil design need to be carefully controlled.

The changes made to the boundary are small and typically have small impact on other physics parameters, Nevertheless, as the design is optimized to provide marginal ballooning stability at full pressure, the changes made to the boundary can potentially de-stabilize the equilibrium with respect to ballooning modes. Future work will augment the target function with various measures of stability. Alternatively, variations in the nullspace of the coupling matrix may be used to optimize the physics properties of the island eliminated equilibrium. Presently, the technique described in this article is being implemented in free boundary PIES calculations where the independent parameters to be varied are those parameters describing the geometry of the coils; the results of which will be described in a future article.

\section{Acknowledgments}

We thank Allen Boozer for discussions, Steve Hirshman for use of the VMEC code, Long-Poe $\mathrm{Ku}$ for assistance implementing numerical codes and performing physics stability analysis, Chris Hegna and Mike Zarnstorff for discussions. 


\section{References}

[1] J.D. Hanson and J.R. Cary. Elimination of stochasticity in stellarators. Phys.Fluids, 27(4):767, 1984.

[2] S.R. Hudson and R.L. Dewar. Manipulation of islands in a heliac vacuum field. Phys.Lett.A, 226:85, 1997.

[3] M.G. Shats, D.L. Rudakov, B.D. Blackwell, L.E. Sharp, R. Tumlos, S.M. Hamberger, and O.I. Fedyanin. Experimental investigation of magnetic stucture of $\mathrm{H} 1$ heliac. Nucl.Fusion, 34(12):1653, 1994.

[4] S.S. Lloyd, H.J. Gardner, T. Hayashi, and S.R. Hudson. Self healing of magnetic islands in a heliac. Journal of Plasma and Fusion Research SERIES, 1:484, 1997.

[5] A.H. Reiman and H.S. Greenside. Calculation of threedimensional MHD equilibria with islands and stochastic regions. Comp.Phys.Commun., 43:157, 1986.

[6] H.S. Greenside, A.H. Reiman, and A. Salas. Convergence properties of a nonvariational $3 \mathrm{~d}$ mhd equlibrium code. J.Comp.Phys., 81:102, 1989.

[7] S.R. Hudson and R.L. Dewar. Analysis of perturbed magnetic fields via construction of nearby integrable field. Phys.Plasmas, 6(5):1532, 1999.

[8] M.C. et.al. Zarnstorff. Physics issues in the design of low aspect-ratio, high $\beta$, quasi-axisymmetric stellarators. $F u$ sion Energy 2000, Procedings of the 18th IAEA Fusion Energy Conference, Sorrento 2000 - to be published, 2000.

[9] Reiman.A. and et.al. Recent advances in the design of quasi-axisymmetric stellarator plasma configurations. Phys. Plasmas, 8, 2001.

[10] S.P. Hirshman and O. Betancourt. Preconditioned descent algorithm for rapid calculations of magnetohydrodynamic equilibria. J.Comp.Phys., 96:99, 1991.

[11] A.J. Lichtenberg and M.A. Lieberman. Regular and Chaotic Dynamics, 2nd ed. Springer-Verlag, New York, 1992.

[12] J.D. Meiss. Symplectic maps, variational principles \& transport. Reviews of Modern Physics, 64(3):795, 1992.

[13] W. H. Press, B. P. Flannery, S. A. Teukolsky, and W. T. Vetterling. Numerical Recipes in Fortran 77: The art of scientific computing. Cambridge University Press, Cambridge, U.K. 


\section{External Distribution}

Plasma Research Laboratory, Australian National University, Australia

Professor I.R. J ones, Flinders University, Australia

Professor J oão Canalle, Instituto de Fisica DEQ/IF - UERJ , Brazil

Mr. Gerson O. Ludwig, Instituto Nacional de Pesquisas, Brazil

Dr. P.H. Sakanaka, Instituto Fisica, Brazil

The Librarian, Culham Laboratory, England

Library, R61, Rutherford Appleton Laboratory, England

Mrs. S.A. Hutchinson, JET Library, England

Professor M.N. Bussac, Ecole Polytechnique, France

Librarian, Max-Planck-Institut für Plasmaphysik, Germany

J olan Moldvai, Reports Library, MTA KFKI-ATKI, Hungary

Dr. P. Kaw, Institute for Plasma Research, India

Ms. P.J . Pathak, Librarian, Insitute for Plasma Research, India

Ms. Clelia De Palo, Associazione EURATOM-ENEA, I taly

Dr. G. Grosso, Instituto di Fisica del Plasma, Italy

Librarian, Naka Fusion Research Establishment, J AERI, J apan

Library, Plasma Physics Laboratory, Kyoto University, J apan

Research Information Center, National Institute for Fusion Science, J apan

Dr. O. Mitarai, Kyushu Tokai University, J apan

Library, Academia Sinica, Institute of Plasma Physics, People's Republic of China

Shih-Tung Tsai, Institute of Physics, Chinese Academy of Sciences, People's Republic of China

Dr. S. Mirnov, TRINITI, Troitsk, Russian Federation, Russia

Dr. V.S. Strelkov, Kurchatov Institute, Russian Federation, Russia

Professor Peter Lukac, Katedra Fyziky Plazmy MFF UK, Mlynska dolina F-2, Komenskeho Univerzita, SK-842 15 Bratislava, Slovakia

Dr. G.S. Lee, Korea Basic Science Institute, South Korea

Mr. Dennis Bruggink, Fusion Library, University of Wisconsin, USA

Institute for Plasma Research, University of Maryland, USA

Librarian, Fusion Energy Division, Oak Ridge National Laboratory, USA

Librarian, Institute of Fusion Studies, University of Texas, USA

Librarian, Magnetic Fusion Program, Lawrence Livermore National Laboratory, USA

Library, General Atomics, USA

Plasma Physics Group, Fusion Energy Research Program, University of California at San Diego, USA

Plasma Physics Library, Columbia University, USA

Alkesh Punjabi, Center for Fusion Research and Training, Hampton University, USA

Dr. W.M. Stacey, Fusion Research Center, Georgia Institute of Technology, USA

Dr. J ohn Willis, U.S. Department of Energy, Office of Fusion Energy Sciences, USA

Mr. Paul H. Wright, Indianapolis, Indiana, USA 
The Princeton Plasma Physics Laboratory is operated by Princeton University under contract with the U.S. Department of Energy.

\author{
Information Services \\ Princeton Plasma Physics Laboratory \\ P.O. Box 451 \\ Princeton, NJ 08543
}

Phone: 609-243-2750

Fax: 609-243-2751

e-mail: pppl_info@pppl.gov

Internet Address: http://www.pppl.gov 